\title{
Application du contrôle actif aux instabilités aérodynamiques
}

\author{
M. SUNYACH et J.C. BÉRA
}

Laboratoire de Mécanique des Fluides et d'Acoustique, URA 263 du CNRS, Ecole Centrale de Lyon, UCB-Lyon I, BP. 163, 69131 Ecully cedex, France

\begin{abstract}
This paper describes experimental investigations in which adaptive methods are applied to various structures of active control of flow instabilities : flow excited cavity oscillations, compressor surge, and bursting phenomena in laminar boundary layer. The main results are as follows : the adaptive controller manages to automatically find its optimal characteristics in order to cancel the instability in the three experiments under consideration. In the boundary layer case, despite the non linear behaviour of the bursting process, a periodic train of artificially generated burst is significantly reduced downstream the cancelling source.
\end{abstract}

\section{INTRODUCTION}

Lorsque les instabilités aérodynamiques sont couplées avec des oscillateurs de type acoustique ou même mécanique, on obtient des oscillations auto-entretenues et les méthodes du contrôle actif acoustique ou vibratoire peuvent s'appliquer. Un nombre significatif de structures de ce type ont été ainsi clairement identifiées et traitées avec succès, du moins en laboratoire. C'est par exemple le cas pour le couplage écoulement/cavité, le couplage acoustique/combustion, certains phénomènes de flutter dans les aubages, le pompage des systèmes de compression et même le décollement tournant.

Une deuxième catégorie d'instabilités liées à la mécanique de l'écoulement lui-même paraissent bien plus complexes. Faute de pouvoir traiter les mécanismes eux-mêmes, on ne peut guère, à l'heure actuelle, envisager que des actions de contrôle local. C'est le cas des écoulements tourbillonnaires, de la transition laminaire-turbulent et des effets de génération de bouffées dans la couche limite établie. Il n'est pas cependant impossible d'agir sur ces phénomènes, car ils restent le plus souvent très sensibles aux conditions aux limites. Nous étudions dans ce qui suit quelques cas d'applications appartenant à ces deux catégories d'instabilité.

\section{I - ALGORITHME AUTO-ADAPTATIF DE CONTROLE D'INSTABILITÉ}

Dans toutes les expériences de contrôle d'instabilité mettant en jeu des modes d'oscillation, un risque d'instabilité artificielle peut résulter du bouclage introduit par le contrôleur lui-même. Dans ce cas, l'emploi de filtres à réponse impulsionnelle infinie paraît le plus indiqué. Les techniques actuelles permettent de rendre ces filtres auto-adaptatifs ce qui leur permet à la fois de réaliser le contrôle optimal, de prendre en compte la variabilité de certains paramètres. De plus, les instabilités artificielles qui peuvent apparaître dans le processus de convergence sont le plus souvent traitées car le rendement acoustique des instabilités et donc le gain à réaliser dans le contrôleur est faible. Le schéma d'un tel contrôleur est donné dans la figure 1 montrant son application au traitement du couplage écoulement/cavité. 
L'algorithme de contrôle prend alors la forme :

$$
\begin{aligned}
& A^{(n+1)}=A^{(n)}+2 k \varepsilon_{n} U^{(n)} \\
& B^{(n+1)}=B^{(n)}+2 k \varepsilon_{n} V^{(n)}
\end{aligned}
$$

Selon le schéma, le filtre D modélisant la source de contrôle est le plus souvent indispensable pour assurer la convergence qui dépend aussi très étroitement du choix du coefficient $k$ et de la longueur des filtres $A$ et $B$.

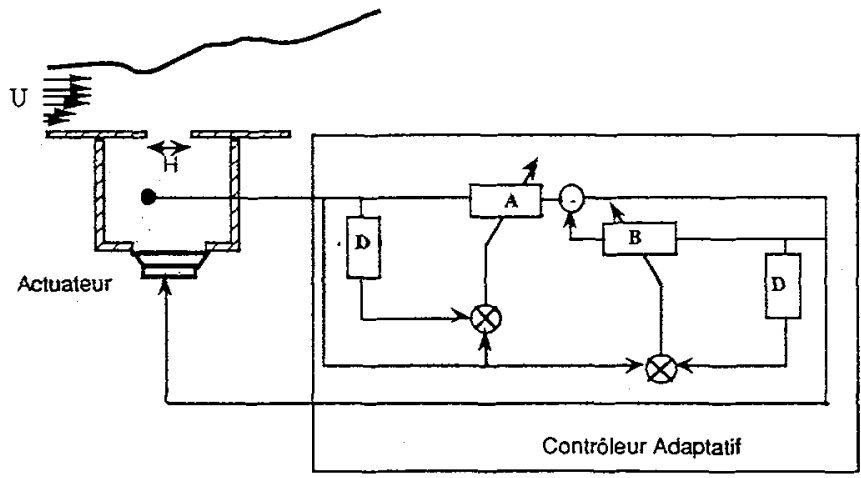

Figure 1.a - Application du contrôle au traitement du couplage écoulement/cavité

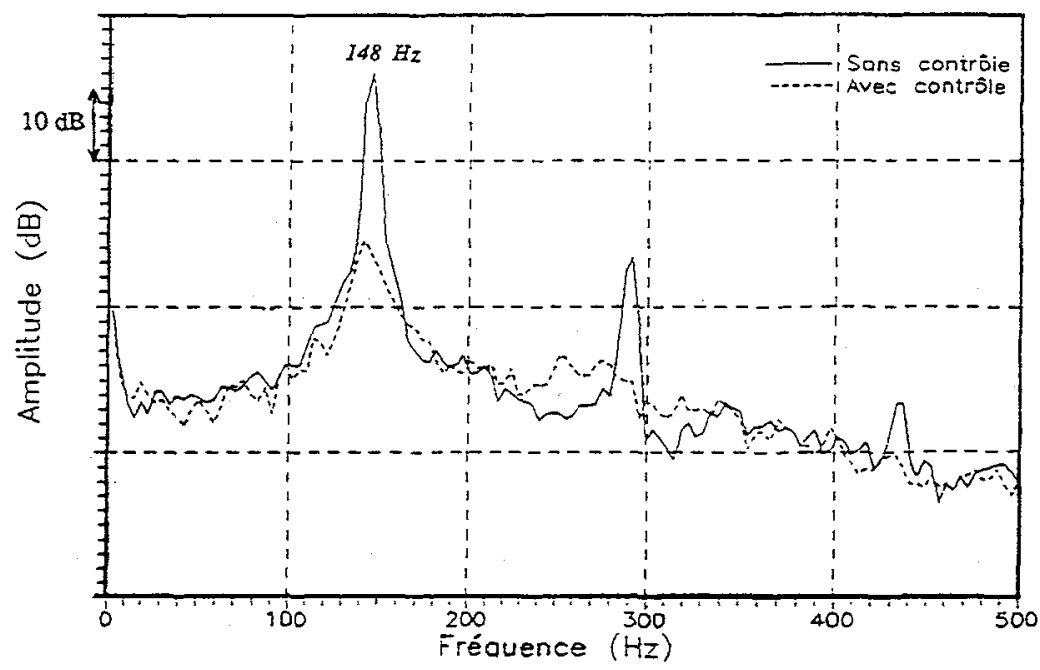

Figure 1.b - Effet du contrôle sur le spectre de pression à l'intérieur de la cavité. (filtre $R I I, N=8, K=0,0015$ ) 


\section{II - APPLICATION AU CONTROLE DES OSCILLATIONS ENTRETENUES PAR LE COUPLAGE ECOULEMENT/CAVITE}

Le schéma de principe étant représenté sur la figure 1 et un modèle simplifié permet de comprendre le mode d'action du contrôle [1]. On peut en effet considérer la cavité comme un oscillateur de Helmholtz, et écrire une équation du type

$$
\frac{d^{r} \xi}{d t^{2}}+\eta_{o} \omega_{o} \frac{d \xi}{d t}+\omega_{o}(1+C(k)+H) \xi=O
$$

dans laquelle $C(k)$ représente le couplage entre les débits "acoustique" et "aérodynamique" traversant le col [2] et $H$ le gain du filtre. Ces termes comprennent des parties imaginaires et l'on observe que leur action se reporte essentiellement sur l'amortissement du système que le contrôle cherche à rendre positif et dans une proportion moindre sur la fréquence de l'oscillation résiduelle du système sous contrôle [3]. Notons que l'intérêt essentiel de ce dispositif par rapport au cas d'un contrôleur statique est sa capacité à traiter des variations continues de la vitesse externe (Figure 2).

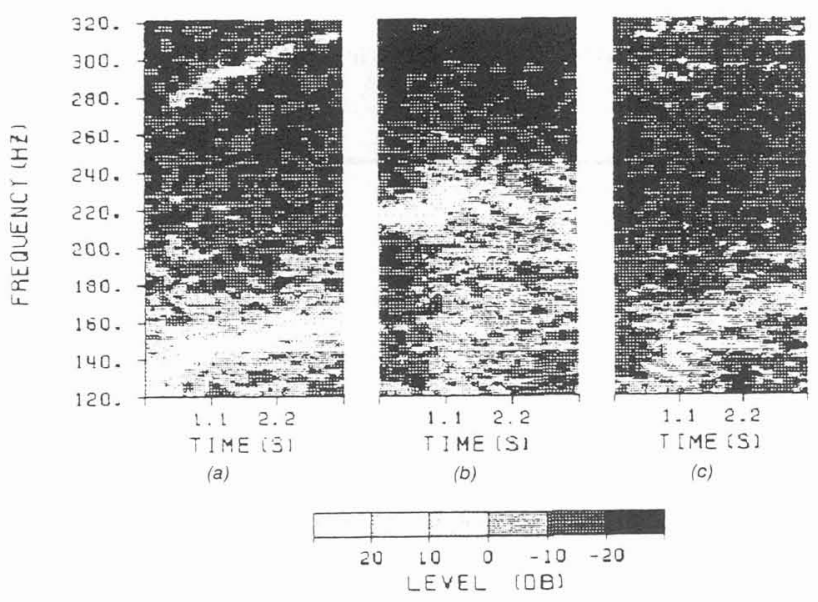

Figure 2 : Représentation t emps-fréquence du niveau de pression :

(a) sans contrôle (fig de gauche),

(b) avec contrôle fixe (fig centrale),

(c) avec contrôle auto-adaptatif (fig de droite).

\section{III - CONTROLE DU POMPAGE D'UN CIRCUIT AERAULIQUE}

Le pompage du système de compresseur (figure 3) est une oscillation auto-entretenue due à l'interaction entre le compresseur et les conduits aérauliques associés. Le comportement du système peut encore être modélisé approximativement par un résonateur de Helmholtz dans lequel le compresseur est la source de perturbation. Comme pour la cavité on peut encore ramener le problème à celui d'un oscillateur à un degré de liberté en supposant un fonctionnement quasi-statique. Introduisant les termes de contrôle dans le modèle de Greitzer [4] on peut encore écrire une équation caractéristique de la forme :

$$
s^{2}+\left[\frac{1}{B T(1+Z)}-\left(B \psi^{\prime}-\mu\right)\right] s+\frac{1}{1+Z}\left[1-\frac{B \psi^{\prime}-\mu}{B T}\right]=O
$$


Cette équation permet de préciser les conditions de stabilité en fonction des pentes des caractéristiques du compresseur et du circuit de décharge $\left(\psi^{\prime}\right.$ et $T$ ) et de la loi de contrôle $Z$. $B$ désigne le paramètre de Greitzer qui est un rapport entre le temps caractéristique du circuit (résonateur) et la fréquence de perturbation liée à la rotation et à la géométrie du compresseur.

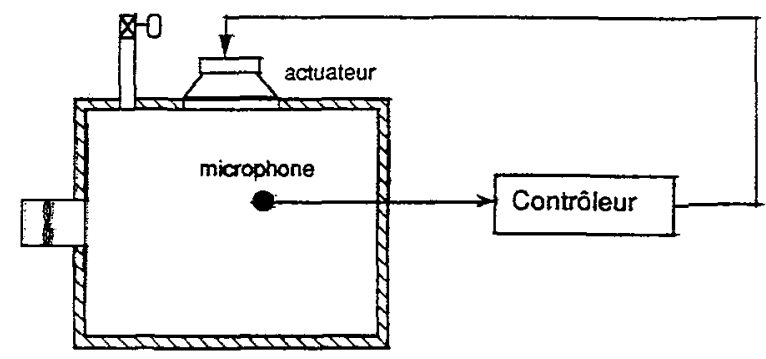

Schéma de principe pour le contrôle du pompage.

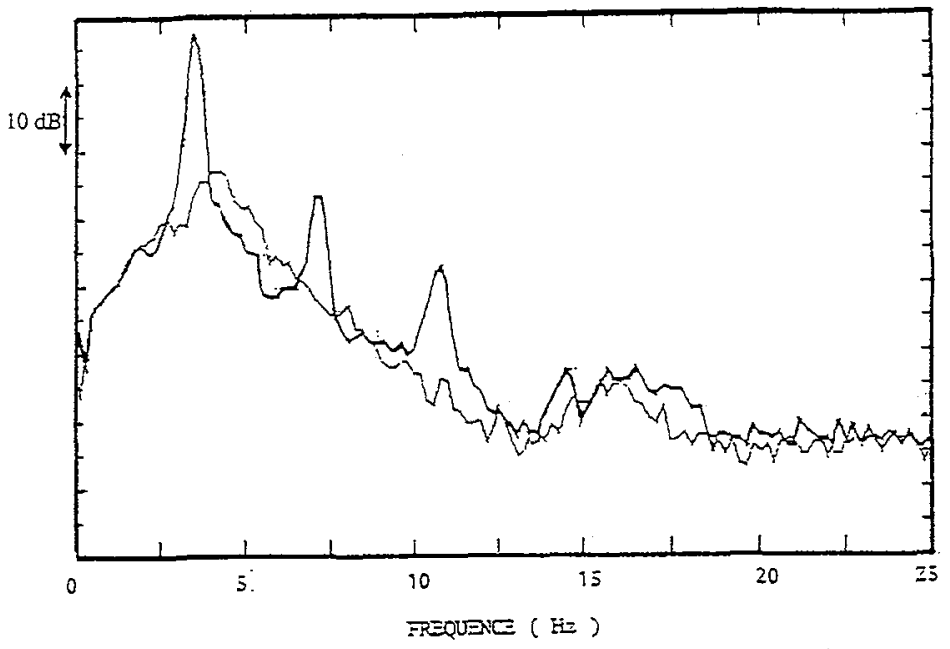

Spectre de la pression à l'intérieur de la capacité sans et avec contrôle ( filtre $R U, N=10, K=0,001$ ).

\section{Figure 3}

Ce modèle peut être validé expérimentalement [3] et permet de retrouver un comportement analogue au cas du couplage écoulement/cavité qui est finalement caractéristique de tous les cas d'oscillations autoentretenues.

Nous faisons toutefois remarquer que le contrôle par l'acoustique n'est pas nécessairement transposable au traitement industriel. Dans les cas pratiques, les méthodes utilisables pour apporter de l'énergie stabilisante ne sont pas toujours linéaires et posent le problème de l'évaluation du module secondaire D dans le contrôleur (Figure 1). 


\section{IV - CONTROLE DE BOUFFEES DE TURBULENCE DANS UNE COUCHE LIMITE LAMINAIRE AU VOISINAGE DE LA TRANSITION}

\section{IV.I Méthode d'essais :}

L'introduction de l'énergie fluctuante ne peut être schématisée de façon aussi simple que dans les cas précédents. La plupart des solutions envisagées pour le contrôle ( $\mathrm{y}$ compris dans les expériences numériques [5] se réduisant à une simple superposition d'un champ contrariant le champ à contrôler. Les expériences effectuées se limitent essentiellement au contrôle de l'onde de Tollmien-Schlichting se développant en régime linéaire et donc au voisinage du bord d'attaque [6].

Pour notre part, nous avons envisagé de créer une bouffée de turbulence dans l'écoulement laminaire puis de le contrôler en utilisant les mêmes méthodes de contrôle que dans les cas d'oscillations autoentretenues. Par soucis de simplicité, nous considérons dans cette expérience préliminaire plutôt un train de bouffées qu'une perturbation isolée.

La figure 4 schématise le dispositif expérimental et montre la signature pariétale de la bouffée créée qui présente une éjection brusque et une zone de "sillage". La figure 5 montre la dispersion du contenu spectral de ces signaux vers une composante large bande lorsqu'on se déplace vers l'aval.

\section{VEINE D'ESSAIS}
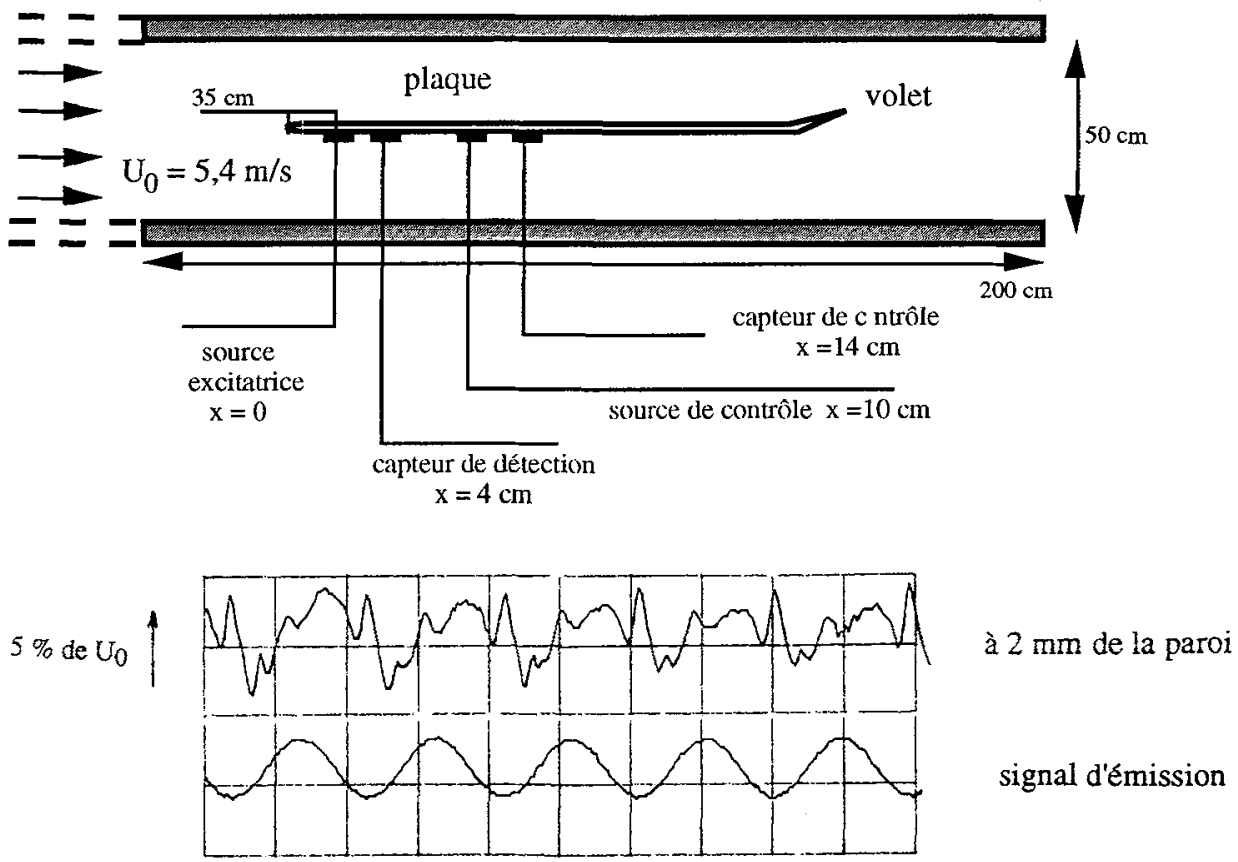

- Dispositif expérimental et signature de la perturbation relevée dans l'écoulement. $\mathrm{x}=5 \mathrm{~cm}$, durée de l'échantillon $50 \mathrm{~ms}$. 


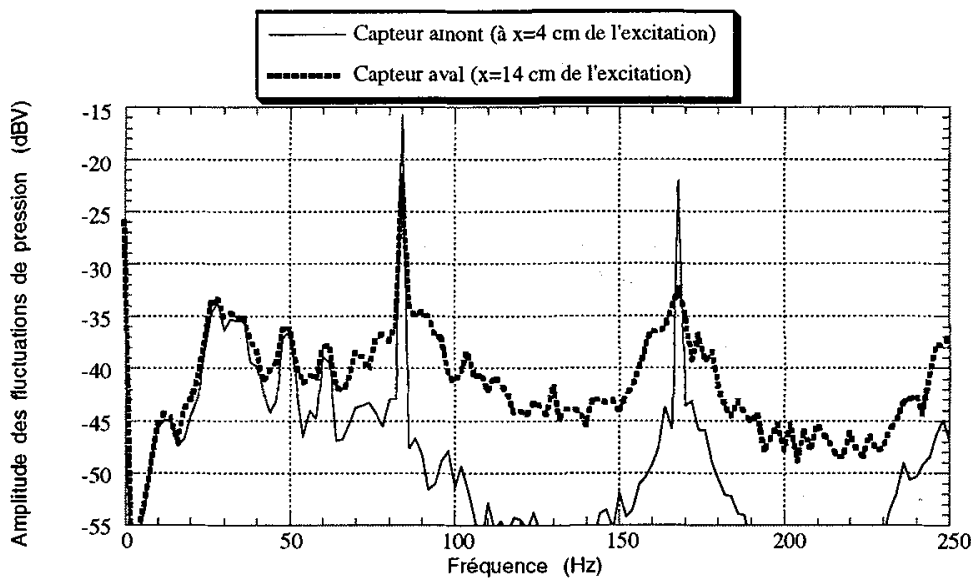

Figure 5 - Spectres des fluctuations de pression en paroi, au niveau des capteurs amont et aval, montrant la création d'une composante large bande.

\section{IV.2 Résultat du contrôle :}

Au niveau du capteur de contrôle, la composante fondamentale et le premier harmonique sont traités. Le comportement de l'écoulement, figure 6, traduit le développement spectral du contrôle mais montre que le générateur et le contrôleur de bouffées doivent être améliorés car ils perturbent encore trop l'écoulement. Il est bien clair que la source de contrôle agit en créant sa propre perturbation et non par superposition linéaire ce qui fait que le passage à la transition paraît s'avancer $(x=12)$ même si le comportement en aval montre des résultats tout-à-fait positifs.

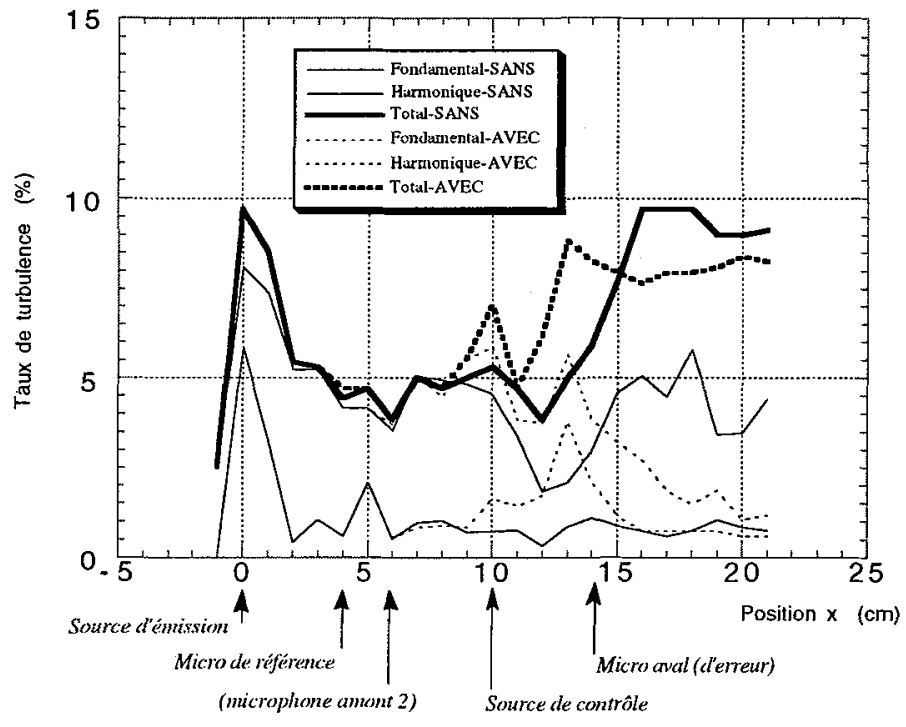

Figure 6 - TURBULENCE avec et sans contrôle, à $1 \mathrm{~mm}$ de la parois. 


\section{$V$ - CONCLUSION}

Le contrôle actif permet de s'opposer aux phénomènes d'oscillations auto entretenues présentes dans les écoulements, et qui résultent de phénoménes de couplage. En plus des progrès qui peuvent être faits dans la compréhension des phénomènes eux-mêmes, il est d'un grand intérêt d'apprendre à traiter les perturbations à la source. Des applications peuvent d'ores et déjà être envisagées. En effet dans de nombreux cas, le contrôle peut s'exercer sur des perturbations déjà installées en raison de leur caractère cyclique et du faible rendement acoustique des perturbations aérodynamiques. Par ailleurs il existe différentes stratégies pour agir sur le bilan de l'énergie fluctuante et de futurs progrès pourront être faits en améliorant notre maîtrise des phénomènes non linéaires..

Pour ce qui est des instabilités de l'écoulement lui même, les premières résultats semblent donner des indications favorables, mais les investigations restent encore du domaine de la recherche.

\section{BIBLIOGRAPHIE:}

[1] MOSER.M. Aktive kontrolle einfacher selbsterregter resonatoren, ACUSTICA, 69, 1989, pp.174-184.

[2] ELDER.S.A.,Force oscillations of a separated shear layer with application to cavity tone effects JASA, 67, 1980, pp.877-890.

[3] SEBBANI.M. Controle actif des instabilités aérodynamiques. Thése Université d'Oran $N^{\circ} 91-19$ (1991).

[4] GREITZER.E.M. Surge and rotating stall in axial flow compressors, Part I: Theoretical compression system model. Trans. ASME Journal of Engineering for power, Vol 98, April 1976, pp 190-198.

[5] MOIN P., KIM.J \& CHOI H., 1989, "On the active control of wall-bounded turbulent flows", AIAA Paper 89-0960.

[6] THOMAS. A.S.W., Viscous Drag Reduction in oundary Layers. Progress in Astronautics and Aeronautics, A.I.A.A, Vol. 123, p. 179-199.

[7] GAD EL HAK M., The Art and Science of flow control. Frontiers in experimental Fluid Mechanics. Lectures Notes in Engineering . $n^{\circ} 46$ Springer Verlag. Berlin 